\title{
Smoothened transduces Hedgehog signal by physically interacting with Costal2/Fused complex through its C-terminal tail
}

\author{
Jianhang Jia, ${ }^{1}$ Chao Tong, ${ }^{1}$ and Jin Jiang ${ }^{1,2,3}$ \\ ${ }^{1}$ Center for Developmental Biology and ${ }^{2}$ Department of Pharmacology, University of Texas Southwestern Medical Center, \\ Dallas, Texas 75390-9133, USA
}

\begin{abstract}
The Hedgehog $(\mathrm{Hh})$ family of secreted proteins controls many aspects of growth and patterning in animal development. The seven-transmembrane protein Smoothened (Smo) transduces the Hh signal in both vertebrates and invertebrates; however, the mechanism of its action remains unknown. We found that Smo lacking its C-terminal tail (C-tail) is inactive, whereas membrane-tethered Smo C-tail has constitutive albeit low levels of Hh signaling activity. Smo physically interacts with Costal2 (Cos2) and Fused (Fu) through its C-tail. Deletion of the Cos2/Fu-binding domain from Smo abolishes its signaling activity. Moreover, overexpressing Cos2 mutants that fail to bind Fu and Ci but retain Smo-binding activity blocks Hh signaling. Taken together, our results suggest that Smo transduces the Hh signal by physically interacting with the Cos2/Fu protein complex.
\end{abstract}

[Keywords: $\mathrm{Smo} ; \mathrm{Hh}$; $\mathrm{Cos} 2 ; \mathrm{Fu}$; signaling; development]

Supplemental material is available at http://www.genesdev.org.

Received July 23, 2003; revised version accepted September 2, 2003.

The Hedgehog (Hh) family of secreted proteins governs cell growth and patterning in numerous developmental processes in both vertebrates and invertebrates (Ingham and McMahon 2001). In addition, misregulation of Hh signaling activity has been implicated in many human disorders including cancers (Villavicencio et al. 2000; Taipale and Beachy 2001). In Drosophila wing development, posterior $(\mathrm{P})$ compartment cells express and secrete $\mathrm{Hh}$ proteins that diffuse into the anterior (A) compartment and induce neighboring A-compartment cells to express decapentaplegic $(d p p)$, which encodes a member of the TGF $\beta / B M P$ family of secreted proteins (Basler and Struhl 1994; Tabata and Kornberg 1994). Dpp then diffuses bidirectionally and functions as a long-range morphogen to control the growth and patterning of cells in the whole wing (Lecuit et al. 1996; Nellen et al. 1996). Although the long-range organizing influence of $\mathrm{Hh}$ is mediated by Dpp, Hh functions as a local morphogen to specify patterns near the A/P compartment boundary by activating other genes including patched (ptc) and engrailed (en) in a concentration-dependent manner (Strigini and Cohen 1997).

${ }^{3}$ Corresponding author.

E-MAIL jin.jiang@utsouthwestern.edu; FAX (214) 648-1960.

Article and publication are at http://www.genesdev.org/cgi/doi/10.1101/ gad.1136603.
Hh exerts its biological influence via a conserved, yet not well-defined, signal transduction pathway (Ingham and McMahon 2001). The reception system for the $\mathrm{Hh}$ signal consists of two multispan transmembrane proteins Patched (Ptc) and Smoothened (Smo). In the absence of $\mathrm{Hh}$, Ptc inhibits Smo activity through a poorly understood mechanism. In Hh-receiving cells, Hh physically interacts with Ptc and alleviates its inhibition on Smo (Chen and Struhl 1996; Stone et al. 1996), allowing Smo to signal downstream. How Smo activates downstream signaling components is a mystery.

In Drosophila, Hh signal transduction culminates in the activation of Cubitus interruptus (Ci), a member of the Gli family of zinc finger transcription factors (Ingham and McMahon 2001). In imaginal disc development, Ci plays dual roles that are performed by two distinct forms. In the absence of $\mathrm{Hh}$, the full-length $\mathrm{Ci}$ (Ci155) undergoes proteolytic processing to generate a truncated form (Ci75) that functions as a repressor to block the expression of Hh-responsive genes such as $d p p$ (AzaBlanc et al. 1997; Methot and Basler 1999). Hh signaling blocks Ci processing to form Ci75. The accumulated Ci155 acts as an activator to turn on other Hh-responsive genes including ptc and en (Alexandre et al. 1996; Methot and Basler 1999).

$\mathrm{Ci}$ processing requires the activities of at least three 
kinases: the cAMP-dependent protein kinase (PKA), GSK3, and CKI (Jiang and Struhl 1998; Y. Chen et al. 1999; Price and Kalderon 1999, 2002; Wang et al. 1999; Jia et al. 2002; Jiang 2002). These kinases phosphorylate $\mathrm{Ci}$ at multiple sites in three clusters in its C-terminal region (Jiang 2002). Hyperphosphorylation of Ci targets it for Slimb/Proteasome-mediated proteolytic processing (Jiang and Struhl 1998; Jiang 2002). Hh appears to induce dephosphorylation of $\mathrm{Ci}$, leading to the blockage of its processing (C.H. Chen et al. 1999).

$\mathrm{Hh}$ regulates $\mathrm{Ci}$ at multiple levels. In addition to blocking its proteolysis, $\mathrm{Hh}$ also induces nuclear translocation of Ci155 and further stimulates its transcriptional activity (Ohlmeyer and Kalderon 1998; C.H. Chen et al. 1999; Wang and Holmgren 1999, 2000; Wang et al. 1999, 2000). In the absence of Hh, Ci155 is retained in the cytoplasm by forming a large protein complex that also includes the kinesin-related protein Costal2 (Cos2), the Ser/Thr kinase Fused (Fu), and the tumor suppressor protein $\mathrm{Su}(\mathrm{fu})$ (Methot and Basler 2000; Wang and Holmgren 2000; Wang et al. 2000). The Ci/Cos2/Fu complex binds microtubules, likely through Cos2, in an Hh-regulated manner (Robbins et al. 1997; Sisson et al. 1997; Stegman et al. 2000). The transcriptional activity of Ci155 appears to be further inhibited by PKA phosphorylation and by stoichiometric interaction with $\mathrm{Su}(\mathrm{fu})$ (Ohlmeyer and Kalderon 1997; Wang et al. 1999). Su(fu) prevents the maturation of Ci155 into a labile hyperactive form, and $\mathrm{Hh}$ alleviates such inhibition through $\mathrm{Fu}$ kinase activity (Ohlmeyer and Kalderon 1997). Su(fu) appears to inhibit Ci155 by both impeding its nuclear translocation and inhibiting its transcriptional activity after it enters the nucleus (Methot and Basler 2000; Wang et al. 2000). One possible mechanism for $\mathrm{Su}(\mathrm{fu})$ to inhibit the transcriptional activator activity of Ci155 is to recruit transcription corepressors (Cheng and Bishop 2002).

Different layers of negative regulation of $\mathrm{Ci}$ appear to be offset by distinct thresholds of Hh signaling activity. Low levels of Hh signaling activity suffice to block $\mathrm{Ci}$ processing but do not stimulate the transcriptional activity of Ci155 (Methot and Basler 1999; Wang and Holmgren 1999; Wang et al. 1999). As a consequence, $d p p$ is derepressed, whereas other genes that are activated by Ci155 remain silent. High levels of $\mathrm{Hh}$ activate $\mathrm{Ci155}$, at least in part, by alleviating $\mathrm{Su}(\mathrm{fu})$-mediated repression (Ohlmeyer and Kalderon 1998). Cos2 was initially identified as a negative component in the Hh pathway; however, a recent study suggested that it also has a positive role in the pathway, as removal of cos 2 function in Hh-receiving cells blocks the transduction of high levels of Hh signaling activity (Wang et al. 2000).

Here we address how Smo relays Hh signal to intracellular signaling components. Although Smo is related to the serpentine family of receptors that transduce signals through trimeric G-proteins (Alcedo et al. 1996; van-den-Heuval and Ingham 1996), no evidence for the involvement of a G-protein in physiological Hh signaling has been obtained (Ingham and McMahon 2001). Here we provide evidence that Smo transduces the Hh signal by physically interacting with the Cos $2 / \mathrm{Fu}$ complex through its C-terminal tail.

\section{Results}

Smo C-tail is essential for its activity

To determine the mechanism by which Smo transduces the Hh signal, we generated epitope-tagged full-length and truncated forms of Smo (summarized in Fig. 1), and assessed their signaling activities in wing imaginal discs using the Gal4/UAS system (Brand and Perrimon 1993). Overexpressing a full-length Smo tagged by GFP at its $\mathrm{N}$ terminus (GFP-Smo) by an MS1096 Gal4 driver resulted in ectopic albeit low levels of Hh pathway activation, as evidenced by the accumulation of high levels of Ci155 and ectopic expression of modest levels of $d p p-l a c Z$ in A-compartment cells away from the A/P compartment boundary (Fig. 2A-A"). Coexpressing Ptc with GFP-Smo inhibited the ectopic $\mathrm{Hh}$ signaling activity caused by overexpressing GFP-Smo alone (Supplementary Fig. 1). These observations suggest that high levels of Smo could at least partially titrate out the inhibitory activity of Ptc, resulting in constitutive $\mathrm{Hh}$ pathway activation. In contrast, overexpressing a truncated form of Smo that lacks the C-tail (GFP-Smo $\Delta \mathrm{CT}$ ) failed to induce ectopic Hh pathway activation (Fig. 2B-B"), even though its level of expression is comparable to that of full-length Smo (Fig. 2 , cf. B and A). Moreover, wing discs expressing multiple copies of UAS-GFP-Smo $\Delta C T$ by MS1096 did not ectopically activate the Hh pathway (data not shown).

We also examined if GFP-Smo $\Delta \mathrm{CT}$ possess any $\mathrm{Hh}$ inducible activity by expressing $U A S-G F P-S m o \Delta C T$ in wing discs carrying a smo mutant clone induced by FLP/ FRT-mediated mitotic recombination $(\mathrm{Xu}$ and Rubin 1993). smo mutant cells expressing GFP-Smo $\Delta$ CT failed to activate $p t c-1 a c Z$ expression near the A/P compartment boundary (Fig. 2D-D"), whereas GFP-Smo could functionally substitute the endogenous Smo to activate ptc-lacZ in A-compartment cells near the A/P border (Fig. 2C-C"). In addition, expressing GFP-Smo but not GFP-Smo $\Delta$ CT in smo zygotic null embryos rescued smo mutant phenotypes (Fig. 2F-H). Similar results were obtained with Myc-tagged full-length (Myc-Smo) and Cterminally truncated Smo (Myc-Smo $\Delta \mathrm{CT}$; Fig. 2I; data not shown). These results demonstrate that the Smo Ctail is essential for its signaling activity.

\section{Membrane-tethered forms of Smo C-tail possess constitutive Hh signaling activity}

To further assess the role of the Smo C-tail in Hh signal transduction, we generated several Flag-tagged Smo deletion mutants that only contain the C-tail portion of Smo (Fig. 1). Sev-SmoCT is a chimeric protein that contains the transmembrane and extracellular domain of Sevenless (Sev) protein fused to SmoCT, whereas MyrSmoCT contains a myristoylation signal at the $\mathrm{N}$ terminus of SmoCT. Surprisingly, A-compartment cells ex- 


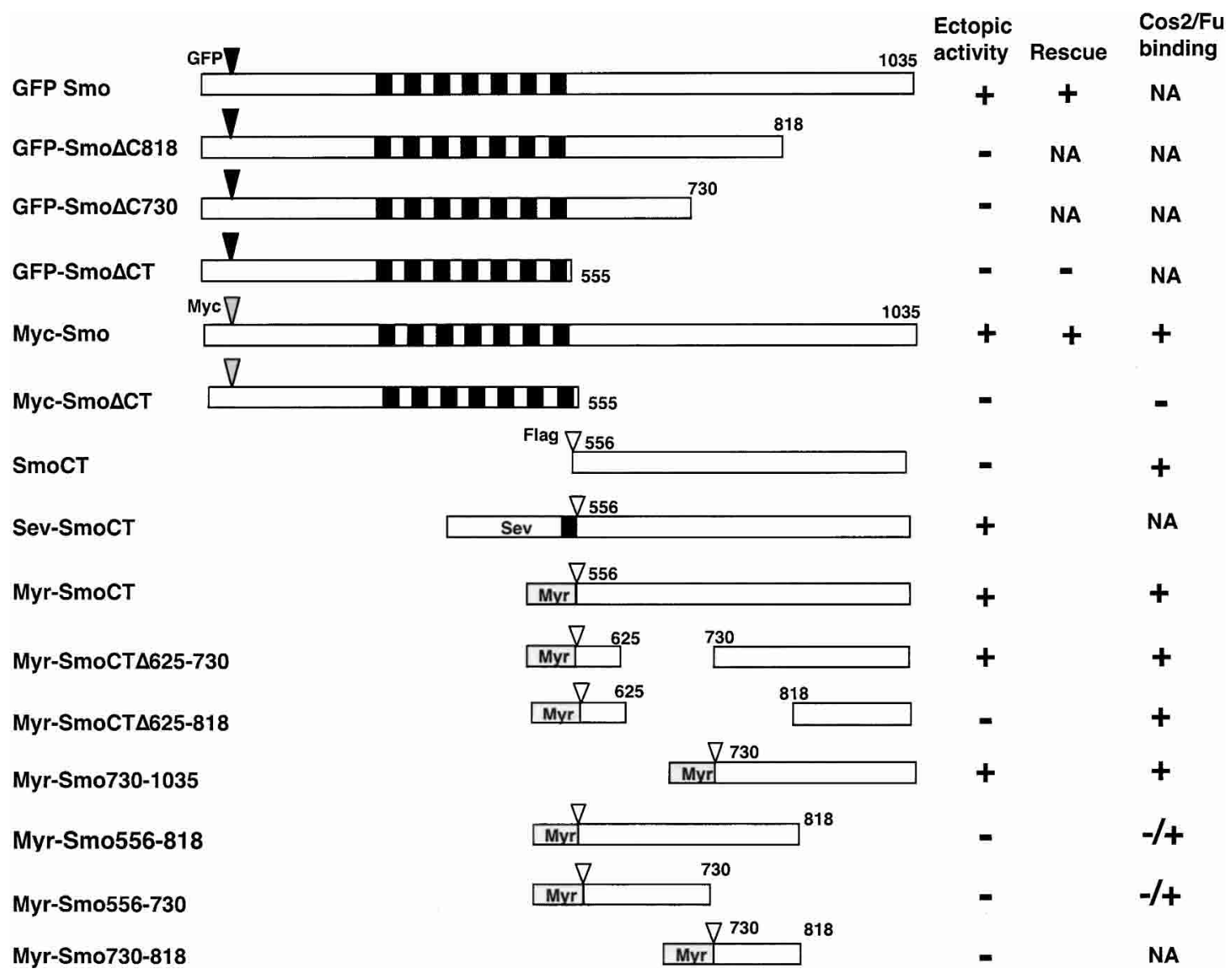

Figure 1. Tagged Smo and its deletion mutants. Filled and gray boxes indicate the transmembrane domains and myristoylation signal (Myr), respectively. Filled, gray, and open triangles indicate the position where GFP, Myc, or Flag tag was inserted, respectively. The amino acid residues that demarcate each deletion mutant are indicated. Individual constructs were assayed for Hh signaling activity by overexpression using the UAS/Gal4 system in developing wing. Ectopic activity was scored when full-length Ci was stabilized and $d p p-l a c Z$ was activated in A-compartment cells away from the A/P compartment boundary. Rescue activity was scored when expressing a given Smo derivative can restore ptc-lacZ in smo mutant clones at the A/P boundary and/or restore naked cuticles in smo zygotic null embryos. Cos2/Fu binding was determined by coimmunoprecipitation. (NA) Not accessed.

pressing either Sev-SmoCT or Myr-SmoCT accumulate high levels of Ci155 and activate $d p p-l a c Z$ in a cell-autonomous manner even when they are distant from the A/P compartment boundary (Fig. 3A- $\mathrm{B}^{\prime \prime}$ ), suggesting that both Sev-SmoCT and Myr-SmoCT can block Ci processing and the formation of Ci75. In contrast, cells expressing untethered SmoCT do not ectopically activate the Hh signaling pathway (data not shown), suggesting that membrane targeting of SmoCT is essential for its activity. As Sev-SmoCT and Myr-SmoCT activate the $\mathrm{Hh}$ signaling pathway to similar extents, we focused our analyses on Myr-SmoCT.

In addition to preventing $\mathrm{Ci}$ processing, $\mathrm{Hh}$ also promotes nuclear translocation of accumulated Ci155 (C.H. Chen et al. 1999; Wang and Holmgren 2000; Wang et al. 2000). To determine whether Myr-SmoCT stimulates Ci155 nuclear translocation, we treated wing discs expressing Myr-SmoCT with Leptomycin B (LMB), an inhibitor that blocks Ci155 nuclear export (C.H. Chen et al. 1999; Wang and Holmgren 2000; Wang et al. 2000). In wild-type wing discs, A-compartment cells near the A/P boundary accumulate Ci155 in the nucleus, whereas cells situated distantly from the $\mathrm{A} / \mathrm{P}$ boundary keep Ci155 in the cytoplasm (Fig. 3C; Wang and Holmgren 2000; Wang et al. 2000). In contrast, A-compartment cells expressing Myr-SmoCT accumulate Ci155 in the nucleus regardless of their locations (Fig. $\left.3 \mathrm{D}, \mathrm{D}^{\prime}\right)$, indicating that Myr-SmoCT stimulates Ci155 nuclear translocation in addition to blocking its processing.

\section{Myr-SmoCT activates Hh signaling independent of endogenous Smo}

To determine if Myr-SmoCT can elicit Hh signaling activity in the absence of endogenous Smo, we misexpressed Myr-SmoCT in wing discs carrying smo-null mutant clones induced by FLP/FRT-mediated mitotic recombination. As shown in Figure 3, smo cells expressing Myr-SmoCT accumulate high levels of $\mathrm{Ci}$ (Fig. $\left.3 \mathrm{E}, \mathrm{E}^{\prime}\right)$, and activate $d p p-l a c Z$ even though they are situ- 

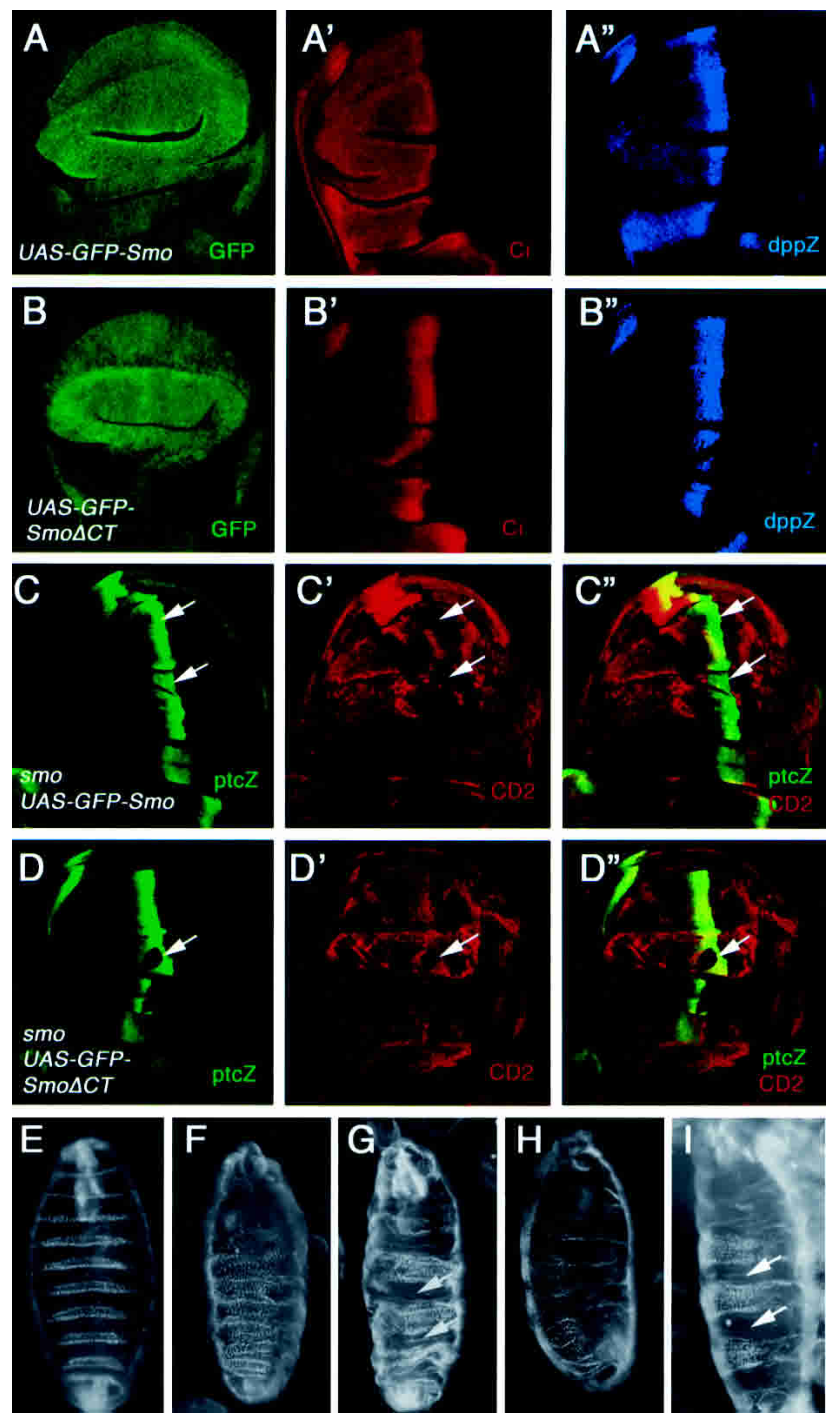

Figure 2. The Smo C-tail is essential for Hh signaling. Wing discs expressing $U A S-G F P$-Smo $\left(A-A^{\prime \prime}\right)$ or $U A S-G F P-S m o \Delta C T$ by $M S 1096\left(B-B^{\prime \prime}\right)$ were immunostained to show the expression of GFP (green in $A, B$ ), Ci155 (red in $A^{\prime}, B^{\prime}$ ), and $d p p-l a c Z$ (blue in $\left.A^{\prime \prime}, B^{\prime \prime}\right)$. Wing discs containing smo mutant clones and expressing UAS-GFP-Smo $\left(C-C^{\prime \prime}\right)$ or UAS-GFP-Smo $D C T$ by MS1096 (D$\left.D^{\prime \prime}\right)$ were immunostained to show the expression of $p t c-l a c Z$ (green) and CD2 (red). Smo mutant clones are recognized by the lack of CD2 expression (arrows). (E-I) Cuticles were prepared from embryos of the following genotypes growing at $18^{\circ} \mathrm{C}$ : wild type $(E), s m o^{3} / s m o^{3}(F), s m o^{3} / s m o^{3}$; prd-Gal4/UAS-GFP-Smo $(G), s m o^{3} / \mathrm{smo}^{3}$; prd-Gal4/UAS-GFP-Smo $\Delta C T(H)$, and $s m o^{3} /$ $s m o^{3}$; prd-Gal4/UAS-Myc-Smo (I). The wild-type embryo exhibits alternate naked cuticles and denticles $(E)$, whereas the $s m o^{3}$ homozygote exhibits the typical zygotic null phenotype with a lawn of denticles covering most of the ventral surface $(F)$. Naked cuticles (indicated by arrows) are restored in alternate segments of $s m o^{3}$ homozygotes expressing either UAS-GFPSmo $(G)$ or UAS-Myc-Smo $(I)$, but not in $s m o^{3}$ homozygotes expressing $U A S-G F P-S m o \Delta C T(H)$.

ated in A-compartment cells distant from the $\mathrm{A} / \mathrm{P}$ boundary (Fig. 3G-G"), suggesting that Myr-SmoCT pos- sesses constitutive Hh signaling activity independent of endogenous Smo. To determine if Myr-SmoCT is refractory to Ptc inhibition, UAS-Ptc and UAS-Myr-SmoCT were co-overexpressed in wing discs by the act > CD2 > Gal4 driver (Pignoni et al. 1997). As shown in Figure 3F, coexpression of Ptc does not block the ectopic Hh signaling activity caused by overexpressing Myr-SmoCT. Hence, Myr-SmoCT appears to escape the inhibitory regulation by Ptc, which explains why it is constitutively active.

\section{Myr-SmoCT does not fully activate the Hh pathway}

Although Myr-SmoCT can stabilize Ci155 and promote its nuclear import, it does not fully stimulate the transcriptional activity of $\mathrm{Ci} 155$, as wing discs expressing $U A S-M y r-S m o C T$ by act $>C D 2>$ Gal4 does not activate $p t c-l a c Z$ and en in most of the A-compartment cells (Fig. 4B; data not shown). Under this condition, ptc-lacZ is only activated at low levels in anteriormost regions of wing discs (Fig. 4B), where cells are more responsive to low levels of Hh signaling activity (Jia et al. 2002).

The inability of Myr-SmoCT to fully activate the Hh pathway is not caused by the lack of enough expression. As a matter of fact, the levels of Myr-SmoCT expressed by act $>C D 2>$ Gal4 is much higher than those of endogenous Smo at the A/P compartment boundary, as determined by immunostaining with an antibody against the Smo C-tail (data not shown). In addition, increasing the dose of Myr-SmoCT by expressing multiple copies of $U A S-M y r-S m o C T$ does not further enhance the Hh signaling activity elicited by Myr-SmoCT (data not shown).

It has been shown that $\mathrm{Su}(\mathrm{fu})$ can inhibit Ci155 activity via a mechanism that is independent of $\mathrm{Ci}$ nuclear localization (Wang et al. 2000). Hence, one possible reason that Myr-SmoCT fails to activate Ci155 is that it does not alleviate the inhibition of Ci155 by $\mathrm{Su}(\mathrm{fu})$. If so, removal of $\mathrm{Su}(\mathrm{fu})$ should allow activation of Ci155 accumulated in Myr-SmoCT-expressing cells. We therefore misexpressed Myr-SmoCT in Su(fu) homozygous wing discs. Although Su(fu) homozygous wing discs do not exhibit any discernible phenotypes (Ohlmeyer and Kalderon 1998), overexpressing Myr-SmoCT in Su(fu) wing discs ectopically activated high levels of ptc-lacZ in most A-compartment cells (Fig. 4C).

\section{Deletion analysis of Smo C-tail}

To further define the region within the Smo C-tail that mediates Hh pathway activation, we generated a series of deletion mutants that remove various parts of the Smo $\mathrm{C}$-tail and examined their abilities to activate the $\mathrm{Hh}$ pathway (Fig. 1). We found that Myr-SmoCT $\Delta 625-730$ and Myr-Smo730-1035 can elicit Hh pathway activation, as indicated by the accumulation of Ci155 and ectopic expression of $d p p-l a c Z$ (Supplementary Fig. 2; Fig. $\left.3 \mathrm{H}_{-} \mathrm{H}^{\prime \prime}\right)$. In contrast, Myr-Smo556-730, Myr-Smo730818, Myr-Smo556-818, and Myr-SmoCT $\Delta 625-818$ do not possess Hh signaling activity (Supplementary Fig. 2; 

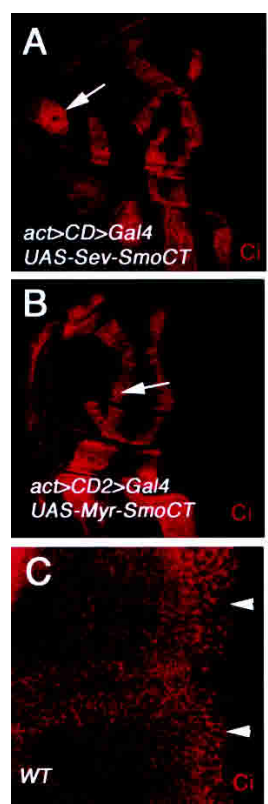
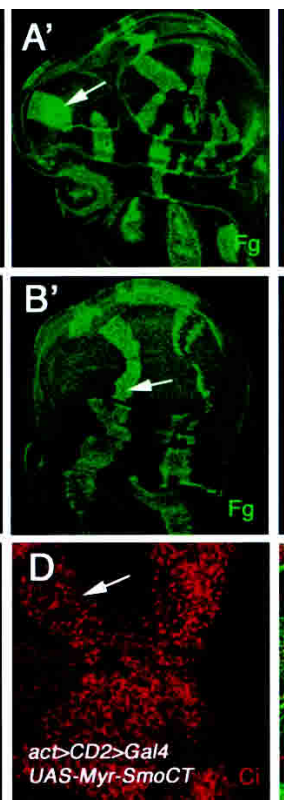
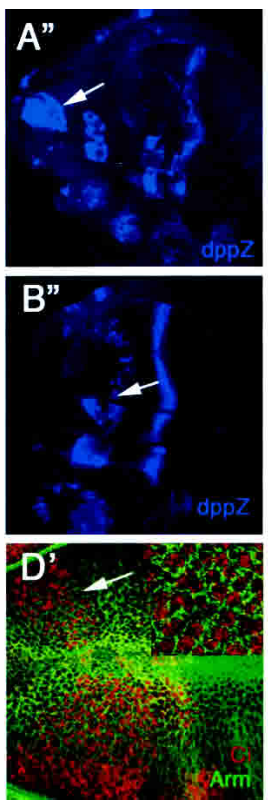
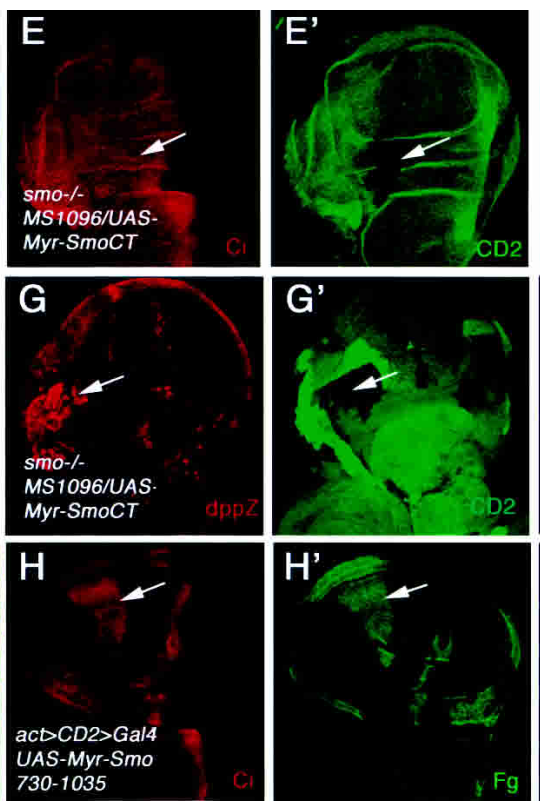
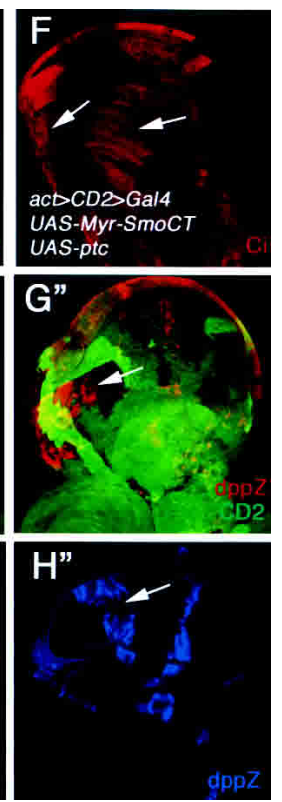

Figure 3. Constitutive Hh signaling activity associated with the Smo C-tail. Wing discs expressing $U A S-S e v-S m o C T\left(A-A^{\prime \prime}\right)$ or $U A S-M y r-S m o C T$ by act $>C D 2>$ Gal4 $\left(B-B^{\prime \prime}\right)$ were stained for Ci155 (red), Flag (green), and $d p p-1 a c Z$ (blue). A-compartment cells expressing either Sev-SmoCT or Myr-SmoCT (recognized by Flag staining) accumulate high levels of Ci155 and activate dpp-lacZ in a cell-autonomous fashion. Wild-type wing disc $(C)$ or wing disc expressing Myr-SmoCT by act $>C D 2>G a 14\left(D-D^{\prime \prime}\right)$ were treated with LMB, followed by immunostaining for Ci155 (red) and Arm (green). In wild-type discs, Ci155 is detected in the nucleus near the A/P border (arrowheads in C). Ci155 translocates into the nucleus in anteriorly situated cells expressing Myr-SmoCT (arrows in $\left.D, D^{\prime}\right)$. The inset in $D^{\prime}$ shows a high-magnification view of anteriorly situated cells expressing Myr-SmoCT. $\left(E, E^{\prime}\right)$ A wing disc carrying an smo mutant clone and expressing MS1096/UAS-Myr-SmoCT was immunostained to show CD2 expression (green) and the accumulation of Ci155 (red). smo mutant cells (marked by the lack of CD2 expression) expressing Myr-SmoCT accumulate high levels of Ci155. (F) A-compartment cells expressing both $U A S-M y r-S m o C T$ and $U A S-p t c$ by act $>C D 2>$ Gal4 accumulate high levels of Ci155 (arrows). Expression from $U A S-p t c$ was confirmed by staining with an anti-Ptc antibody (data not shown). (G-G') A wing disc expressing MS1096/UAS-Myr-SmoCT and containing $s m o^{3}$ clones was immunostained to show the expression of $d p p-1 a c Z$ (red) and CD2 (green). $s m o^{3}$ mutant cells are marked by the lack of CD2 expression. Overexpressing Myr-SmoCT activates $d p p-l a c Z$ in $s m o^{3}$ mutant cells (arrows). $\left(H-H^{\prime \prime}\right)$ A wing disc expressing UAS-Myr-Smo730-1035 under the control of act > CD2 > Gal4 was stained for Ci155 (red), Flag (green), and $d p p-1 a c Z$ (blue). A-compartment cells expressing Myr-Smo730-1035 accumulate high levels of Ci155 and activate dpp-lacZ.

data not shown). Deleting the C-terminal sequence from full-length Smo (GFP-Smo $\Delta 818$ and GFP-Smo $\Delta$ C730) also abolishes Smo activity as overexpressing these deletion mutants failed to ectopically activate $d p p-l a c Z$ (Supplementary Fig. 2; data not shown). These results suggest that the C-terminal half of the Smo C-tail (amino acids $730-1035$ ) is critical for Smo activity.

\section{Smo physically interacts with Cos2/Fu through its C-tail}

Interestingly, expressing Myr-SmoCT at high levels inhibits the expression of endogenous $p t c$ and en near the A/P compartment boundary (Fig. 4D-D"), suggesting that Myr-SmoCT can interfere with the ability of endogenous Smo to transduce high levels of Hh signaling activity. The inhibition of $p t c$ and en expression by MyrSmoCT can be suppressed by coexpressing GFP-Smo (Fig. 4E-E"). One hypothesis that accounts for these observations is that Myr-SmoCT may compete with endogenous Smo for a downstream signaling effector(s).
Several known intracellular Hh signaling components, including $\mathrm{Cos} 2, \mathrm{Fu}$, and $\mathrm{Ci}$, form large protein complexes (Robbins et al. 1997; Sisson et al. 1997). As no signaling intermediates between Smo and the Cos2 complex have been identified, we sought to determine if Smo transduces Hh signal by physically interacting with the Cos 2 complex. To facilitate the detection of complex formation, we transfected S2 cells with DNA constructs expressing Myc-tagged full-length Smo (Myc-Smo) or its C-tail deletion mutant (Myc-Smo $\Delta \mathrm{CT}$ ), HA-tagged Cos2, $\mathrm{Fu}$, and $\mathrm{Ci}$. Cell extracts were immunoprecipitated with a mouse $\alpha$ Myc antibody, followed by Western blot analysis with $\alpha \operatorname{Cos} 2, \alpha \mathrm{Fu}$, or $\alpha \mathrm{Ci}(2 \mathrm{~A})$ antibody. Myc-Smo but not Myc-Smo $\Delta \mathrm{CT}$ pulled down Cos2 and Fu (Fig. 5B), suggesting that Myc-Smo binds the Cos $2 / \mathrm{Fu}$ complex and the Smo C-tail is essential for this interaction. $\mathrm{Ci}$ was not consistently pulled down by Myc-Smo (data not shown). It is possible that $\mathrm{Ci} / \mathrm{Smo}$ interaction is very dynamic. For example, Ci may dissociate from the Cos2/ Fu complex after the complex binds Smo. We also found that Myc-Smo expressed in S2 cells can pull down en- 

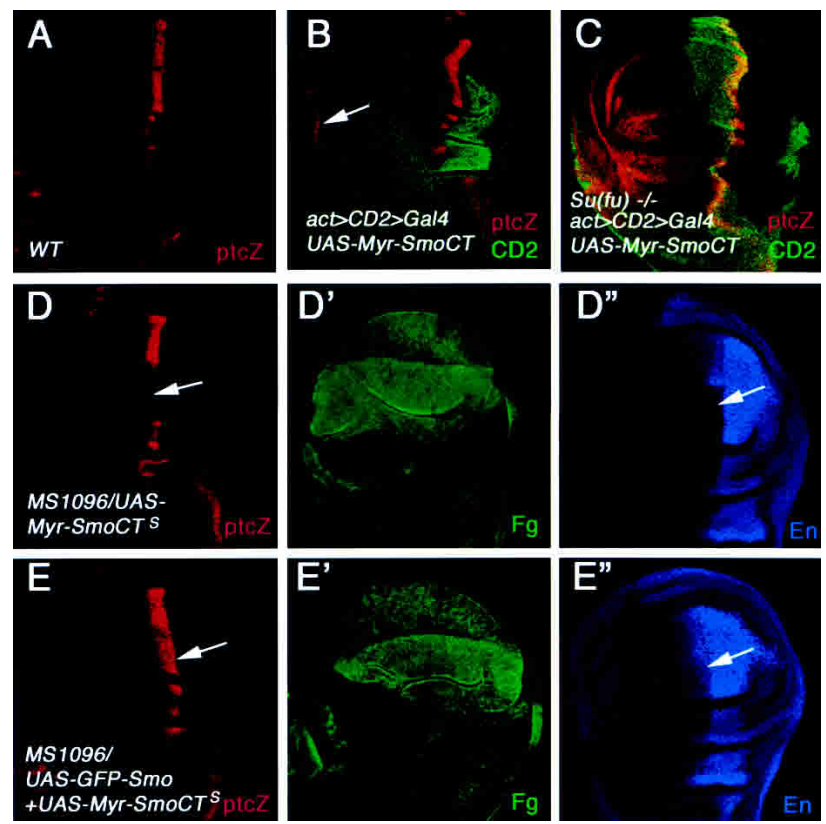

Figure 4. Myr-SmoCT does not fully activate the Hh pathway and interferes with endogenous Smo. (A) ptc-lacZ expression in a wild-type wing disc. Wild-type $(B)$ or $S u(f u)^{L P}$ homozygous $(C)$ wing discs expressing UAS-Myr-SmoCT by act >CD2 > Gal4 were immunostained to show the expression of CD2 (green) and ptc-lacZ (red). Myr-SmoCT-expressing cells are recognized by the lack of CD2 staining. Myc-SmoCT only activates low levels of ptc-lacZ in anteriormost cells (arrow in $B$ ). $(C)$ In contrast, most A-compartment cells expressing Myr-SmoCT activate high levels of ptc-lacZ in Su(fu) homozygous discs. $\left(D-E^{\prime \prime}\right)$ Wing disc expressing a strong line of Myr-SmoCT (UAS-Myr$\left.S m o C T^{S}\right)$ alone $\left(D-D^{\prime \prime}\right)$ or in conjunction with GFP-Smo $\left(E-E^{\prime \prime}\right)$ were immunostained to show the expression of ptc-lacZ (red), Flag (green), and En (blue). High levels of Myr-SmoCT inhibit the expression of $p t c-l a c Z$ and en in A-compartment cells near the A/P boundary (arrows in $D, D^{\prime \prime}$ ), which is reversed by coexpressing GFP-Smo (arrows in $E, E^{\prime \prime}$ ).

dogenous Cos2 and Fu (Fig. 5C). As S2 cells do not express $\mathrm{Ci}$, this result indicates that $\mathrm{Smo}$ can interact with $\mathrm{Cos} 2$ and $\mathrm{Fu}$ independently of $\mathrm{Ci}$.

To determine if Smo binds Cos2 and $\mathrm{Fu}$ in vivo, we expressed Myc-Smo or Myc-Smo $\Delta \mathrm{CT}$ in wing discs by the MS1096 Gal4 driver. Wing disc extracts were immunoprecipitated with the $\alpha$ Myc antibody, followed by Western blot analysis with $\alpha \operatorname{Cos} 2, \alpha \mathrm{Fu}$, and $\alpha \mathrm{Ci}$ antibodies. As shown in Figure 5D, Myc-Smo but not MycSmo $\Delta$ CT pulled down Cos2 and Fu. Myc-Smo did not pull down detectable levels of $\mathrm{Ci}$ (data not shown). We also coexpressed $U A S-H h$ with $U A S-M y c-S m o$ to determine if the interaction between Myc-Smo and Cos $2 / \mathrm{Fu}$ is affected. As expected, ectopic expression of Hh stabilizes coexpressed Myc-Smo, as more Myc-Smo was pulled down by the $\alpha$ Myc antibody (Fig. 5D). However, the amounts of Cos2 and Fu pulled down by Myc-Smo only increase modestly (Fig. 5D), suggesting that overexpressing Myc-Smo alone may already saturate Cos $2 / \mathrm{Fu}$ binding. Interestingly, a significant fraction of $\mathrm{Fu}$ bound to Myc-Smo became phosphorylated when $\mathrm{Hh}$ was co- expressed, as indicated by its mobility shift (Fig. 5D). Hence, overexpressed Myc-Smo binds Cos2/Fu in vivo, but appears to stimulate $\mathrm{Fu}$ phosphorylation only when $\mathrm{Hh}$ is coexpressed.

When expressed in S2 cells, both Myr-SmoCT and Myr-Smo730-1035 pulled down endogenous Cos2/Fu (Fig. 5E, lane 2; data not shown), suggesting that SmoCT is sufficient for binding to the Cos $2 / \mathrm{Fu}$ complex. Consistent with their inability to activate the Hh pathway, both Myr-Smo556-730 and Myr-Smo556-818 pull down diminishing levels of Cos $2 / \mathrm{Fu}$ (Fig. 5E, lanes 3,4). Intriguingly, Myr-SmoCT $\Delta 625-818$, which does not possess $\mathrm{Hh}$ signaling activity, appears to bind Cos2/Fu with affinity similar to that of Myr-SmoCT (Fig. 5E, lane 5). In addition, the untethered SmoCT also binds Cos2/Fu (Fig. $5 \mathrm{E}$, lane 1). These observations suggest that binding of Smo to Cos $2 / F u$ per se does not trigger Hh pathway activation. It seems that membrane association and the Smo sequence between amino acids 730 and 818 are also critical for SmoCT to activate the Hh pathway.

To define the Cos 2 domain(s) that mediates Smo binding, we generated a series of HA-tagged Cos2 deletion mutants (summarized in Fig. 5A) and examined their ability to bind Myc-Smo in S2 cells by coimmunoprecipitation. It appears that Myc-Smo binds Cos2 through at least two regions of Cos2: the microtubule-binding domain and the C-tail, as HA-Cos2MB, HA-Cos2CT1, and HA-Cos2CT2 were pulled down robustly by MycSmo (Fig. 5F, lanes 5,6,8). HA-Cos $2 \Delta \mathrm{N} 2$ also binds strongly to Myc-Smo (Fig. 5F, lane 3). In contrast, HACos2CC was not pulled down by Myc-Smo (Fig. 5F, lane 4). Intriguingly, relatively less HA-Cos2, HA-Cos $2 \Delta \mathrm{N} 1$, and HA-Cos $2 \Delta \mathrm{C}$ were pulled down by Myc-Smo (Fig. $5 \mathrm{~F}$, lanes 1,2$)$. One possibility is that Smo-binding domains in these large proteins could be partially "masked." Alternatively, they might be unstable when bound to Smo in the absence of Fu. Consistent with the latter hypothesis, we found that coexpression of $\mathrm{Fu}$ with HA-Cos2 dramatically increases the amount of HA-Cos2 pulled down by Myc-Smo (data not shown).

\section{Blockage of Hh signal transduction by Cos2 deletion mutants}

To access the physiological significance of Smo/Cos 2 interaction in Hh signal transduction, we expressed Cos2 mutants that lack Ci- and Fu-binding domains but retain Smo-binding activity in wing discs and examined their effects on Hh signaling. We reasoned if Smo transduces $\mathrm{Hh}$ signal by recruiting the Cos $2 / \mathrm{Fu}$ complex, overexpressing such Cos2 deletion mutants should titrate out endogenous Smo and prevent it from interacting with the endogenous Cos2/Fu complex. As a consequence, Hh signal transduction should be blocked. The Ci- and Fubinding domains have been mapped to the $\mathrm{N}$-terminal half of Cos2 (Fig. 5A; Monnier et al. 2002), and Cos $2 \Delta \mathrm{N} 2$ failed to bind $\mathrm{Ci}$ and $\mathrm{Fu}$ in yeast (G. Wang and J. Jiang, unpubl.). As shown in Figure 6A, overexpressing $\operatorname{Cos} 2 \Delta \mathrm{N} 2$ in wing discs blocks Hh signaling, as indicated by the loss of $p t c-l a c Z$. The inhibition of $\mathrm{Hh}$ signaling 

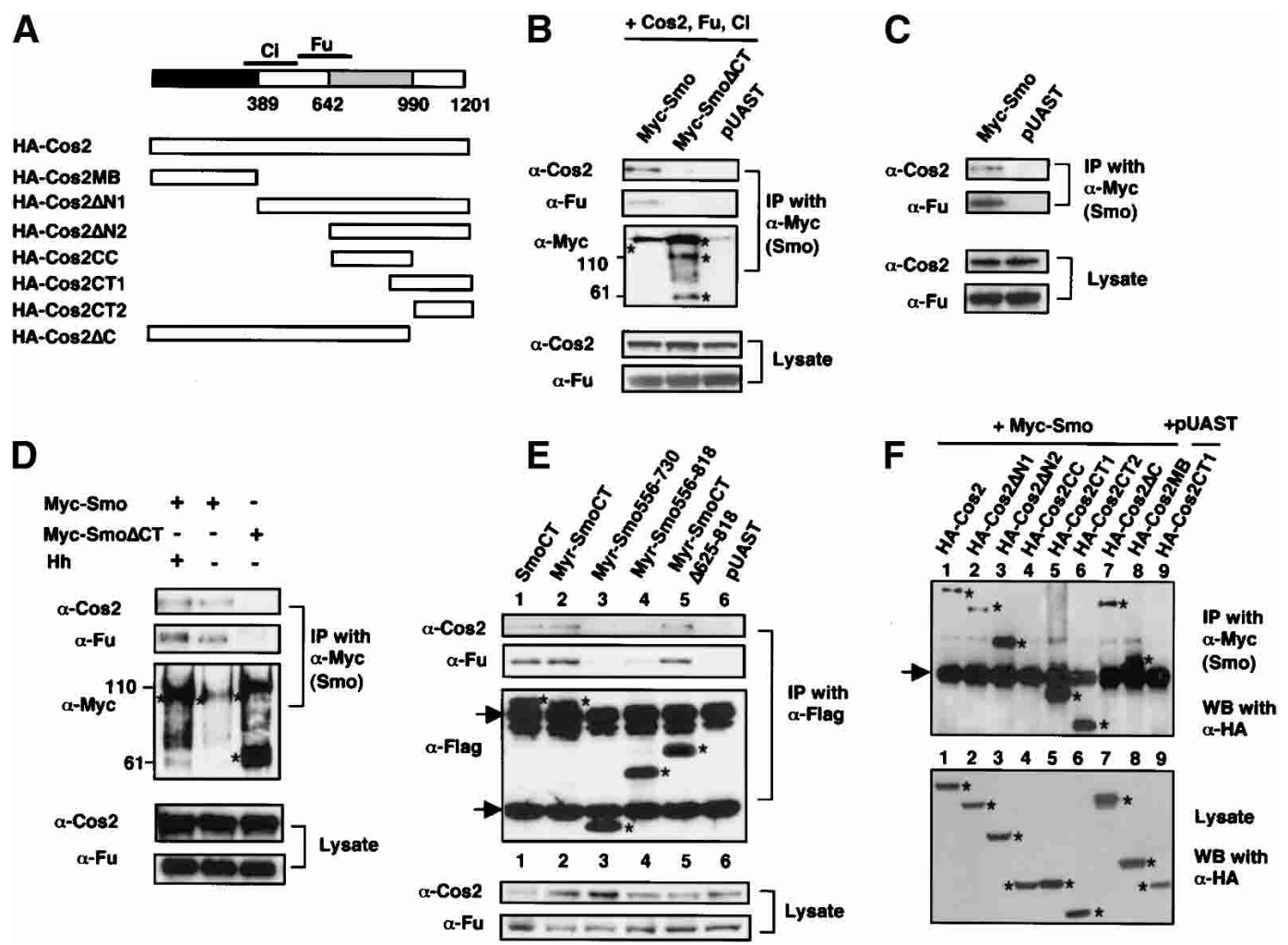

Figure 5. Smo binds to the Cos2/Fu complex through its C-tail. (A) HA-tagged Cos2 and its deletion mutants. The microtubulebinding $(\mathrm{MB})$ and coiled-coil $(\mathrm{CC})$ domains are indicated by the black and gray boxes, respectively. The Ci- and Fu-binding domains are demarcated by lines above the diagram. $(B, C)$ S2 cells were transfected with Myc-Smo or Myc-Smo $\Delta$ CT expressing constructs with $(B)$ or without $(C)$ Cos2-, Fu-, and Ci-expressing constructs. The blank expressing vector pUAST was used as a control. Cell extracts were immunoprecipitated (IP) with anti-Myc antibody. Immunoprecipitates and $5 \%$ of cell lysates were analyzed by immunoblotting (WB) with indicated antibodies. Myc-Smo but not Myc-SmosCT pulled down overexpressed as well as endogenous Cos2 and Fu. Of note, overexpressed Myc-Smo and Myc-Smo $\Delta \mathrm{CT}$ exhibit slow mobility (indicated by asterisks). A similar observation was made with overexpressed vertebrate Smo (Stone et al. 1996). (D) Cell extracts were prepared from 400 wing discs expressing UAS-Myc-Smo, $U A S-M y c-S m o \Delta C T$, or $U A S-M y c-S m o$ in conjunction with $U A S-H h$ under the control of MS1096. Wing disc extracts were immunoprecipitated with anti-Myc antibody, followed by immunoblotting with indicated antibodies. Five percent of lysates were analyzed by direct Western with Cos2 or Fu antibody. Myc-Smo but not Myc-Smo $\Delta$ CT pulled down endogenous Cos 2 and Fu. Ectopic Hh appears to stimulate phosphorylation of Fu bound to Myc-Smo, as indicated by slower mobility. Myc-Smo and Myc-Smo $\Delta \mathrm{CT}$ are indicated by asterisks. $(E)$ S2 cells were transfected with the indicated Flag-tagged Smo constructs. Cell lysates were immunoprecipitated with anti-Flag antibody followed by immunoblotting with anti-Cos2 (top), anti-Fu (middle), or anti-Flag (bottom) antibodies. Asterisks indicate the position of proteins expressed from corresponding Smo constructs. Arrows indicate IgG. $(F)$ S2 cells were transfected with Myc-Smo in conjunction with various HA-tagged Cos2 deletion mutants. Cell extracts were immunoprecipitated with anti-Myc antibody, followed by immunoblotting with anti-HA antibody. Five percent of cell lysates were also subjected to Western blotting with anti-HA antibody (bottom). Asterisks indicate the position of HA-tagged Cos2 proteins expressed from corresponding constructs (bottom) or immunoprecipitated with Myc-Smo (top). Of note, HA-Cos2MB immunoprecipitated with Myc-Smo runs very closely to IgG.

activity by $\operatorname{Cos} 2 \Delta \mathrm{N} 2$ correlates with Smo binding as Cos2CC, which does not bind Smo, fails to block Hh signaling (Fig. 6B). Moreover, overexpressing the Cos2 C-tail (Cos2CT1) also results in pathway inhibition, as manifested by the reduction of ptc-lac $Z$ and anterior en expression (Fig. 6D-D") as well as fu-like wing phenotypes (Fig. 6F). If the inhibition of Hh signaling by Cos2 deletion mutants is caused by titrating out endogenous Smo and preventing it from binding to endogenous Cos $2 /$ Fu complex, then increasing the amounts of Smo should reverse such a blockage. Indeed, coexpressing GFP-Smo with $\operatorname{Cos} 2 \Delta \mathrm{N} 2$ restores $p t c-l a c Z$ expression (Fig. $6 \mathrm{C}-\mathrm{C}^{\prime \prime}$ ).

\section{Discussion}

The seven-transmembrane protein Smo plays a central role in transducing Hh signal. In this study, we demonstrated that the Smo C-terminal tail (C-tail) is essential for its function. Surprisingly, we found that the Smo Ctail possesses constitutive albeit low levels of signaling activity when it is tethered to plasma membrane. We provided biochemical evidence that Smo interacts with the Cos2/Fu complex through its C-tail, and Smo/Cos2 interaction is mediated at least in part through the Cterminal region of Cos2. In addition, we provided evidence that Smo/Cos2 interaction is essential for Hh sig- 

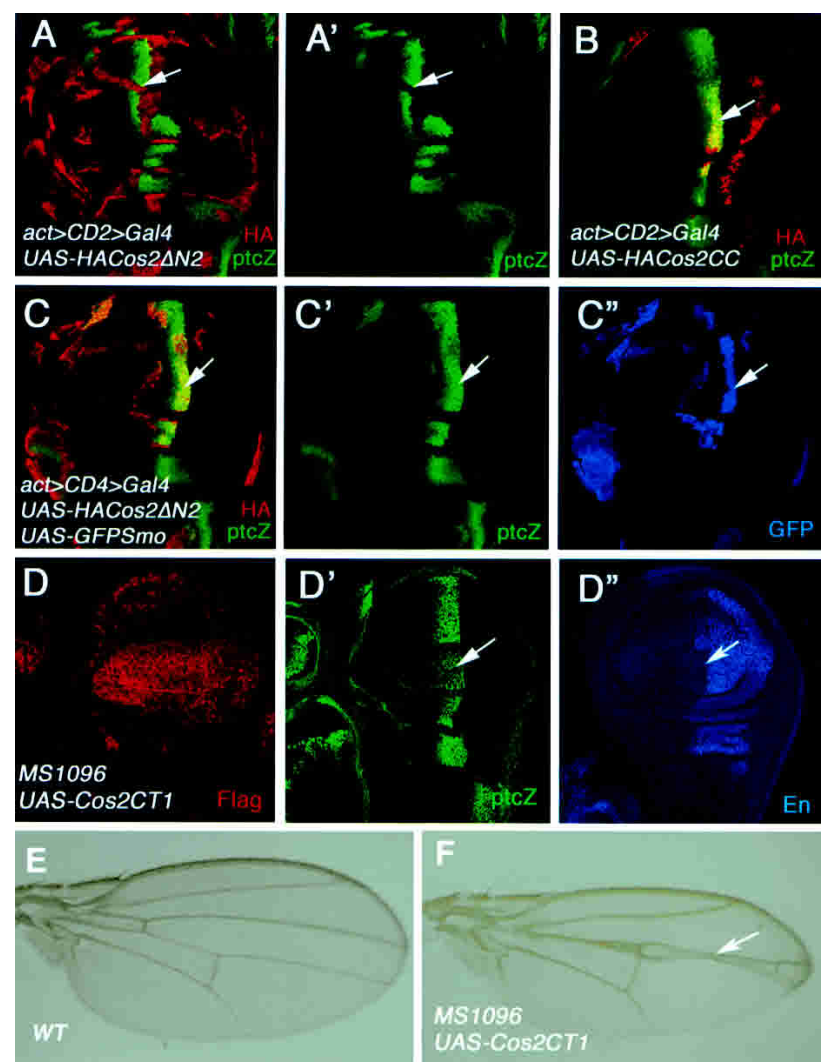

Figure 6. Blockage of $\mathrm{Hh}$ signaling by $\operatorname{Cos} 2$ deletion mutants. $\left(A, A^{\prime}\right)$ A wing disc expressing UAS-HA-Cos $2 \Delta N 2$ by $a c t>C D 2>$ Gal4 was immunostained for ptc-lacZ (green) and $\mathrm{HA}$ (red). Cos $2 \Delta \mathrm{N} 2$ blocks the expression of ptc-lac $Z$ near the $\mathrm{A} / \mathrm{P}$ border (arrows). (B) A wing disc expressing UAS-HACos2CC by act $>C D 2>$ Gal4. Cos2CC failed to suppress ptclac $Z$ expression at the $\mathrm{A} / \mathrm{P}$ compartment boundary (arrows). ( $C-$ $\left.C^{\prime \prime}\right)$ A wing disc expressing both UAS-HA-Cos2 $\triangle N 2$ and UASGFP-Smo by act >CD2 > Gal4 was immunostained to show the expression of HA (red), ptc-lacZ (green), and GFP (blue). Coexpression of GFP-Smo with Cos $2 \Delta \mathrm{N} 2$ restores the expression of ptc-lacZ near the A/P border (arrows). $\left(D-D^{\prime \prime}\right)$ A wing disc expressing UAS-Flag-Cos2CT1 by MS1096 was stained to show the expression of Flag (red), ptc-lacZ (green), and En (blue). High levels of Cos2CT1 suppress the expression of ptc-lacZ and en (arrows). A wild-type adult wing (E) or adult wing expressing UAS-Flag-Cos2CT1 by MS1096 (F). Overexpressing Cos2CT1 results in wing phenotypes similar to those caused by the $\mathrm{fu}$ mutation.

nal transduction. Our results suggest that Smo transduces $\mathrm{Hh}$ signal by directly recruiting the $\operatorname{Cos} 2 / \mathrm{Fu}$ complex to the plasma membrane rather than by activating a signaling cascade involving a secondary messenger, a mechanism ascribed to most members of the serpentine receptor family.

\section{Smo transduces Hh signal through its C-tail}

A previous structure-function study on mammalian Smo using cultured cells suggested that the Smo C-tail is not essential for Smo activity (Murone et al. 1999). Here we showed that Smo deletion mutants lacking the Smo C-tail $(\mathrm{Smo} \Delta \mathrm{CT})$ or part of it $(\mathrm{Smo} \Delta \mathrm{C} 818$ and Smo $\Delta$ C730) are inactive when overexpressed in wing discs. Moreover, Smo $\Delta \mathrm{CT}$ does not substitute endogenous Smo to transduce signal in Hh-receiving cells. Hence, the Smo C-tail is absolutely required for Smo activity. It is not clear what causes the discrepancy. One possibility is that Drosophila Smo may act differently from mammalian Smo. Alternatively, Smo may behave differently in vivo versus in vitro. Consistent with this, we found that mouse Smo without a C-tail fails to induce ventral markers in chick spinal cords, whereas a control full-length Smo does (W. Zhang, R. Lu, and J. Jiang, unpubl.).

Perhaps a more surprising finding of our study is that the Smo C-tail suffices to induce Hh pathway activation. We found that overexpressing the membrane-tethered Smo C-tail (Myr-SmoCT, Sev-SmoCT) blocks Ci processing, induces $d p p-l a c Z$ expression, and stimulates nuclear translocation of Ci155. Myr-SmoCT is refractory to Ptc inhibition and activates Hh-pathway independent of endogenous Smo. Membrane tethering appears to be crucial for the Smo C-tail to activate the Hh pathway, as untethered SmoCT has no signaling activity. This is consistent with previous observations that cell surface accumulation of Smo correlates with its activity (Denef et al. 2000; Zhu et al. 2003).

Although the Smo C-tail has constitutive Hh signaling activity, it does not possess all the activities associated with full-length Smo. For example, overexpressing MyrSmoCT in A-compartment cells away from the A/P compartment boundary does not significantly activate $p t c$ and en, which are normally induced by high levels of $\mathrm{Hh}$. In addition, Myr-SmoCT cannot substitute endogenous Smo at the A/P compartment boundary to transduce high levels of Hh signaling activity, as boundary smo mutant cells expressing Myr-SmoCT failed to express $p t c$ in response to Hh (data not shown).

The failure of the Smo C-tail to transduce high $\mathrm{Hh}$ signaling activity is due to its inability to antagonize $\mathrm{Su}(\mathrm{fu})$. Although Myr-SmoCT blocks Ci processing to generate Ci75, the activity of Ci155 accumulated in Myr-SmoCT-expressing cells is still blocked by $\mathrm{Su}(\mathrm{fu})$, as removal of $\mathrm{Su}(\mathrm{fu}$ ) function from Myr-SmoCT-expressing cells allows Ci155 to activate ptc to high levels. As Myr-SmoCT stimulates nuclear translocation of Ci155, the inhibition of Ci155 by Su(fu) in Myr-SmoCT-expressing cells must rely on a mechanism that is independent of impeding Ci nuclear translocation.

\section{Physical interaction between Smo and Cos2/Fu}

Several observations prompted us to determine whether Smo could transduce Hh signal by physically interacting with the Cos2/Fu complex. First, although Smo is related to G-protein-coupled receptors, no genetic or pharmacological evidence has been obtained to support the involvement of a G-protein in a physiological Hh signaling process (Ingham and McMahon 2001). Second, MyrSmoCT can interfere with the ability of endogenous Smo 
to transduce high levels of Hh signaling activity, which can be offset by increasing the amount of full-length Smo. This implies that Myr-SmoCT may compete with full-length Smo for binding to limiting amounts of downstream signaling components (Fig. 7E,F). Third, extensive genetic screens failed to identify $\mathrm{Hh}$ signaling components that may link Smo to the Cos $2 / \mathrm{Fu}$ complex (Amanai and Jiang 2001; Vegh and Basler 2003).

Using a coimmunoprecipitation assay, we demonstrated that Smo interacts with the Cos $2 / \mathrm{Fu}$ complex both in S2 cells and in wing imaginal discs, and the Smo C-tail appears to be both necessary and sufficient to mediate this interaction. We narrowed down the Cos2/Fubinding domain to the C-terminal half of the Smo C-tail (between amino acids 818 and 1035). Furthermore, we found that both the microtubule-binding domain (amino acids 1-389) and the C-terminal tail (amino acids 9901201) of Cos2 interact with Smo. As none of these Cos2 domains binds $\mathrm{Fu}$, this implies that the Cos2/Smo interaction is not mediated through $\mathrm{Fu}$. $\mathrm{Ci}$ is also dispensable for $\mathrm{Smo} / \mathrm{Cos} 2 / \mathrm{Fu}$ interaction, as Smo binds $\mathrm{Cos} 2 / \mathrm{Fu}$ in
$\mathrm{S} 2$ cells in which $\mathrm{Ci}$ is not expressed (Aza-Blanc et al. 2000). However, our results did not rule out the possibility that Smo could interact with the Cos2/Fu complex through multiple contacts. For example, Smo could simultaneously contact Cos2 and Fu. Nor did we demonstrate that binding of Cos 2 to Smo is direct. Indeed, we failed to detect direct protein-protein interaction between Smo and Cos2 in yeast (data not shown). It is possible that a bridging molecule(s) is required to link Smo to the Cos2/Fu complex. Alternatively, Smo needs to be modified in vivo in order to bind Cos2. It has been shown that Hh stimulates phosphorylation of Smo (Denef et al. 2000); hence, it is possible that phosphorylation of Smo might be essential for recruiting the Cos $2 / \mathrm{Fu}$ complex.

Several lines of evidence suggest that $\mathrm{Smo} / \mathrm{Cos} 2 / \mathrm{Fu}$ interaction is important for $\mathrm{Hh}$ signal transduction. (1) Deletion of the Cos2-binding domain from Smo, either in the context of full-length Smo or the Smo C-tail, abolishes Smo signaling activity. (2) Overexpressing Cos 2 deletion mutants that no longer bind $\mathrm{Fu}$ and $\mathrm{Ci}$ but retain
A
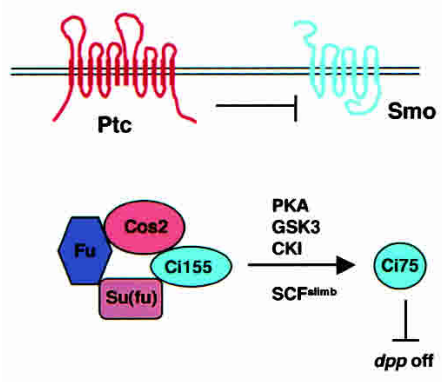

D
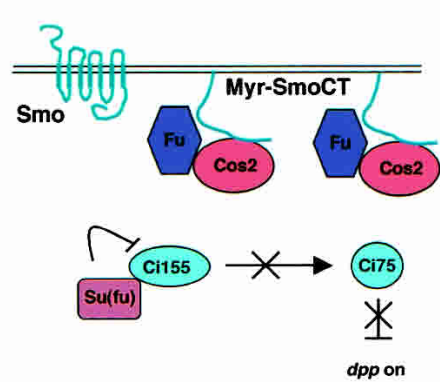

B

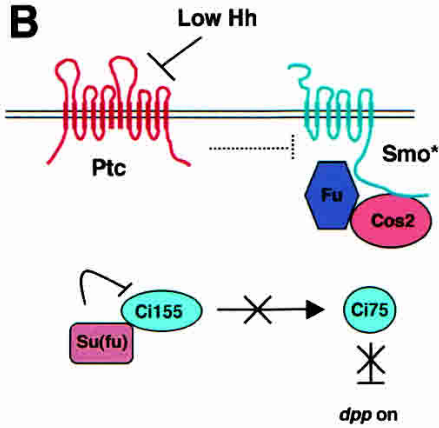

$\mathbf{E}$

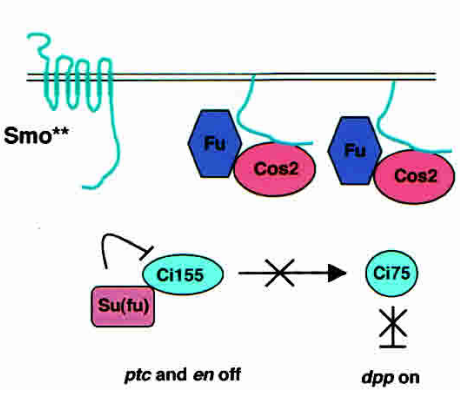

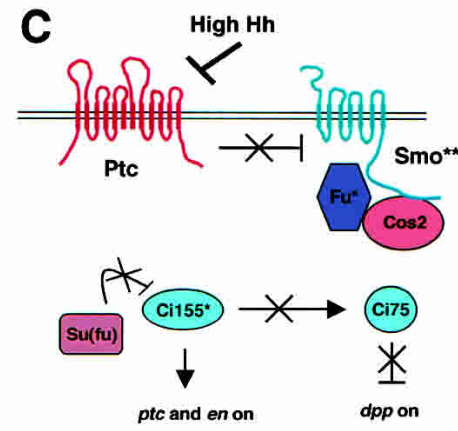

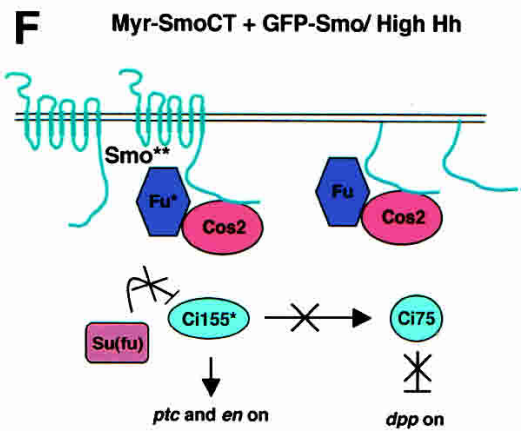

Figure 7. Signaling by Smo and its C-tail in response to different thresholds of Hh. $(A)$ In the absence of Hh, Ptc prevents cell surface accumulation of Smo. In addition, the Smo C-tail may adopt a "closed" conformation that prevents it from binding to Cos2/Fu. Inside the cell, the full-length $\mathrm{Ci}$ (Ci155) forms a complex with $\mathrm{Cos} 2, \mathrm{Fu}$, and $\mathrm{Su}(\mathrm{fu})$. The majority of Ci155 undergoes proteolytic processing to generate the repressor form (Ci75). Ci processing also requires the activity of PKA, GSK3, CKI, and SCF slimb (Jiang 2002). (B) The inhibition of Smo by Ptc is partially alleviated by low levels of Hh (indicated by broken line), leading to an increase of Smo on the cell surface. In addition, the Smo C-tail may adopt an "open" conformation, which allows Smo to bind the Cos2/Fu complex and inhibit its Ci-processing activity. Under this condition, Ci75 is not produced and $d p p$ is derepressed. However, the transcriptional activity of Ci155 is still suppressed by $\mathrm{Su}(\mathrm{fu}) .(C)$ High levels of Hh completely block Ptc, resulting in a further increase in Smo signaling activity. Hyperactive forms of Smo (indicated by two asterisks) stimulate the phosphorylation and activity of bound Fu (indicated by asterisk), which in turn antagonizes $\mathrm{Su}(\mathrm{fu})$ to activate Ci155, leading to the expression of $p t c$ and $e n$. $(D)$ Overexpressed membrane-tethered SmoCT binds the Cos2/Fu complex and inhibits Ci processing independently of Hh and endogenous Smo. However, the activity of Ci155 is still blocked by Su(fu). (E) In the presence of Hh, overexpressed Myr-SmoCT competes with activated Smo for binding to $\operatorname{Cos} 2 / \mathrm{Fu}$ and prevents it from activating $\mathrm{Fu} .(F)$ Increasing the amount of full-length Smo by overexpressing GFP-Smo restores $\mathrm{Smo}^{\star \star} / \mathrm{Cos} 2 / \mathrm{Fu}$ interaction, allowing $\mathrm{Smo}^{\star \star}$ to activate $\mathrm{Fu}$, which in turn stimulates Ci155. 
a Smo-binding domain intercept Hh signal transduction. We previously provided genetic evidence that Cos 2 has a positive role in transducing $\mathrm{Hh}$ signal in addition to its negative influence on the Hh pathway, as Ci155 is no longer stimulated into labile and hyperactivity forms by high levels of Hh in cos2 mutant cells (Wang et al. 2000). In light of our finding that Smo interacts with Cos $2 / \mathrm{Fu}$, the simplest interpretation for a positive role of Cos 2 is that it recruits $\mathrm{Fu}$ to $\mathrm{Smo}$ and allows $\mathrm{Fu}$ to be activated by $\mathrm{Smo}$ in response to $\mathrm{Hh}$.

Of note, interaction between SmoCT and Cos2/Fu per se is not sufficient for triggering Hh pathway activation. For example, Myr-SmoCT $\Delta 625-818$, which binds Cos2/ $\mathrm{Fu}$ to the same extent as Myr-SmoCT, does not possess Hh signaling activity. The fact that Myr-Smo $\Delta$ CT625730 and Myr-Smo730-1035 can activate the Hh pathway suggests that Smo sequence between amino acids 730 and 818 is essential. This domain may recruit factors other than $\operatorname{Cos} 2 / \mathrm{Fu}$ to achieve Hh pathway activation. Alternatively, it might target SmoCT to an appropriate signaling environment.

\section{Implications for threshold responses to $\mathrm{Hh}$}

An important property of Hh family members in development is that they can elicit distinct biological responses via different concentrations. How different thresholds of Hh signal are transduced by Smo to generate distinct transcriptional outputs is not understood. Our results suggest that Smo can function as a molecular sensor that converts quantitatively different $\mathrm{Hh}$ signals into qualitatively distinct outputs (Fig. 7).

In the absence of $\mathrm{Hh}$, the cell surface levels of Smo are low (Denef et al. 2000; Zhu et al. 2003). In addition, the Smo C-tail may adopt a "closed" conformation that prevents it from binding to Cos2/Fu (Fig. 7A). Low levels of Hh partially inhibit Ptc, leading to an increase of Smo on the cell surface. In addition, the Smo C-tail may adopt an "open" conformation, which allows Smo to bind the Cos2/Fu complex and inhibit its Ci-processing activity (Fig. 7B). Low levels of Hh signaling activity can be mimicked by overexpression of either full-length Smo or membrane-tethered forms of the Smo C-tail (Fig. 7D). High levels of Hh completely inhibit Ptc, resulting in a further increase in Smo signaling activity. Hyperactive Smo stimulates the phosphorylation and activity of bound $\mathrm{Fu}$, which in turn antagonizes $\mathrm{Su}(\mathrm{fu})$ to activate Ci155 (Fig. 7C). Consistent with this, we found that $\mathrm{Fu}$ bound to Myc-Smo became phosphorylated in response to ectopic Hh.

The Smo sequence $\mathrm{N}$ terminus to SmoCT (SmoN) appears to be essential for conferring high Smo activities. It is not clear how SmoN modulates the activity of SmoCT. SmoN might recruit additional effector(s) or target SmoCT to a microdomain with a more favorable signaling environment. Alternatively, SmoN might function as a dimerization domain that facilitates interaction between two SmoCTs, as in the case of receptor tyrosine kinases (Schlessinger 2000). It is also not clear how Smo/ $\mathrm{Cos} 2 / \mathrm{Fu}$ interaction inhibits $\mathrm{Ci}$ processing. One possi- bility is that Smo/Cos2 interaction may cause disassembly of the Cos2/Ci complex, which could prevent $\mathrm{Ci}$ from being hyperphosphorylated, as Cos $2 / \mathrm{Ci}$ complex formation might be essential for targeting $\mathrm{Ci}$ to its kinases. Consistent with this view, we found that $\mathrm{Ci}$ is barely detectable in the Cos $2 /$ Fu complex bound to Smo.

Physical association of the receptor complex with a downstream signaling component has also been demonstrated for the canonic Wnt pathway whereby the Wnt coreceptor LRP-5 interacts with Axin, a molecular scaffold in the Wnt pathway (Mao et al. 2001). Hence, Hh and Wnt/Wg pathways appear to use a similar mechanism to transmit signal downstream of their receptor complexes.

\section{Materials and methods}

\section{Mutants and transgenes}

$\mathrm{Su}(\mathrm{fu})^{L P}$ and $s m o^{3}$ are null alleles (Preat 1992; Chen and Struhl 1998). MS1096, act > CD2 > Gal4, prd-Gal4, UAS-ptc, UAS$h h, d p p-1 a c Z$, and ptc-lacZ have been described (Pignoni et al. 1997; Chen and Struhl 1998; Wang et al. 1999). To construct $U A S-G F P$-Smo, the coding sequence for GFP was amplified by PCR and inserted into the SfiI site (amino acid 35) of smo cDNA. The resulting fusion gene was subcloned into a pUAST vector between the NotI and XhoI sites. GFP-Smo $\Delta$ CT, GFPSmo $\Delta 730$, and GFP-Smo $\Delta 818$ were derived from GFP-Smo by introducing a stop codon at amino acids 555, 730, and 818, respectively. Myc-Smo and Myc-Smo $\Delta \mathrm{CT}$ contain six copies of Myc-tag inserted at amino acid 35. To construct UASFlagSmoCT, smo cDNA encoding the C-terminal region of Smo from amino acids 556 to 1035 was amplified by PCR and subcloned in the BgIII and XhoI sites of a pUAST-Flag vector. To tether SmoCT to the membrane, a myristoylation signal from Src (MGNKCCSKRQ) or the Sevenless transmembrane and extracellular domains (Struhl and Adachi 1998) were inserted at the $\mathrm{N}$ terminus of Flag-SmoCT to generate UAS-Myr-SmoCT or Sev-SmoCT. Membrane-tethered deletion mutants of SmoCT were generated by a PCR-based approach using appropriate primers. HA-Cos2 contained two copies of HA tags at the $\mathrm{N}$ terminus of Cos2 (Wang et al. 2000). HACos $2 \Delta \mathrm{C}$ was derived from HA-Cos2 by introducing a stop codon after amino acid 993. HA-Cos $2 \Delta \mathrm{N} 1, \mathrm{HA}-\operatorname{Cos} 2 \mathrm{~N} 2$, HA-Cos2CT1, and HACos2CT2 contain two copies of HA tag fused N-terminal to amino acids 389, 642, 906, and 991, respectively. HA-Cos2CC was derived from HA-Cos $2 \Delta \mathrm{N} 2$ by introducing a stop codon after amino acid 993. Flag-Cos2CT1 contains one copy of Flag tag $\mathrm{N}$-terminal to amino acid 906. Transformants were generated using standard P-element-mediated transformation. Multiple independent transformant lines were generated and examined for each construct.

Cell culture, transfection, immunoprecipitation, and Western blot analysis

S2 cells were cultured in the Schneider's Drosophila Medium (Invitrogen) with $10 \%$ fetal bovine serum, $100 \mathrm{U} / \mathrm{mL}$ penicillin, and $100 \mu \mathrm{g} / \mathrm{mL}$ streptomycin. Transfection was carried out using the Calcium Phosphate Transfection Kit (Specialty Media) according to the manufacturer's instructions. Immunoprecipitation and Western blot analyses were performed using standard protocols as previously described (Robbins et al. 1997; C.H. 
Chen et al. 1999). The antibodies used were mouse $\alpha$ Myc, 9E10 and $\alpha \mathrm{HA}, \mathrm{F} 7$ (Santa Cruz); mouse $\alpha$ Flag, M2 (Sigma); mouse $\alpha \operatorname{Cos} 2$ and rabbit $\alpha \mathrm{Fu}$ (Ascano et al. 2002); and rat $\alpha \mathrm{Ci}, 2 \mathrm{~A}$ (Motzny and Holmgren 1995).

\section{Immunostaining of imaginal discs}

Standard protocols for immunofluorescence staining of imaginal discs were used (Jiang and Struhl 1995). LMB treatment of imaginal disc was done as described (Wang et al. 2000). The antibodies used in this study were rat $\alpha \mathrm{Ci}, 2 \mathrm{~A}$ (Motzny and Holmgren 1995), rabbit anti- $\beta$ Gal (Cappel), mouse $\alpha$ CD2 (Serotec), rabbit $\alpha$ GFP (Clonetech), and mouse $\alpha$ En and Arm (DSHB, Iowa University).

\section{Acknowledgments}

We thank Bing Wang and Liping Luo for technical assistance, and Gelin Wang and Kazuhito Amanai for initial experiments on Smo/Cos2 interaction. We thank Drs. D. Robbins, M. Scott, S. Cohen, P. Beachy, and G. Struhl for antibodies and fly stocks. This work was supported by grants from the NIH, the Searle Scholar Program from Chicago Trustee, the Leukemia and Lymphoma Society Scholar Program, and the Endowed Scholar Program in Biomedical Science from UT Southwestern Medical Center to J. Jiang.

The publication costs of this article were defrayed in part by payment of page charges. This article must therefore be hereby marked "advertisement" in accordance with 18 USC section 1734 solely to indicate this fact.

\section{References}

Alcedo, J., Ayzenzon, M., Von Ohlen, T., Noll, M., and Hooper, J.E. 1996. The Drosophila smoothened gene encodes a sevenpass membrane protein, a putative receptor for the Hedgehog signal. Cell 86: 221-232.

Alexandre, C., Jacinto, A., and Ingham, P.W. 1996. Transcriptional activation of Hedgehog target genes in Drosophila is mediated directly by the Cubitus interruptus protein, a member of the GLI family of the zinc finger DNA-binding proteins. Genes \& Dev. 10: 2003-2013.

Amanai, K. and Jiang, J. 2001. Distinct roles of Central missing and Dispatched in sending the Hedgehog signal. Development 128: 5119-5127.

Ascano Jr., M., Nybakken, K.E., Sosinski, J., Stegman, M.A., and Robbins, D.J. 2002. The carboxyl-terminal domain of the protein kinase fused can function as a dominant inhibitor of hedgehog signaling. Mol. Cell. Biol. 22: 1555-1566.

Aza-Blanc, P., Ramirez-Weber, F., Laget, M., Schwartz, C., and Kornberg, T. 1997. Proteolysis that is inhibited by Hedgehog targets Cubitus interruptus protein to the nucleus and converts it to a repressor. Cell 89: 1043-1053.

Aza-Blanc, P., Lin, H.Y., Ruiz i Altaba, A., and Kornberg, T.B. 2000. Expression of the vertebrate Gli proteins in Drosophila reveals a distribution of activator and repressor activities. Development 127: 4293-4301.

Basler, K. and Struhl, G. 1994. Compartment boundaries and the control of Drosophila limb pattern by hedgehog protein. $\mathrm{Na}$ ture 368: 208-214.

Brand, A.H. and Perrimon, N. 1993. Targeted gene expression as a means of altering cell fates and generating dominant phenotypes. Development 118: 401-415.

Chen, Y. and Struhl, G. 1996. Dual roles for patched in sequestering and transducing Hedgehog. Cell 87: 553-563.
1998. In vivo evidence that Patched and Smoothened constitute distinct binding and transducing components of a Hedgehog receptor complex. Development 125: 4943-4948.

Chen, C.H., von Kessler, D.P., Park, W., Wang, B., Ma, Y., and Beachy, P.A. 1999. Nuclear trafficking of Cubitus interruptus in the transcriptional regulation of Hedgehog target gene expression. Cell 98: 305-316.

Chen, Y., Cardinaux, J.R., Goodman, R.H., and Smolik, S.M. 1999. Mutants of cubitus interruptus that are independent of PKA regulation are independent of hedgehog signaling. Development 126: 3607-3616.

Cheng, S.Y. and Bishop, J.M. 2002. Suppressor of Fused represses Gli-mediated transcription by recruiting the SAP18-mSin3 corepressor complex. Proc. Natl. Acad. Sci. 99: 5442-5447.

Denef, N., Neubuser, D., Perez, L., and Cohen, S.M. 2000. Hedgehog induces opposite changes in turnover and subcellular localization of patched and smoothened. Cell 102: 521531.

Ingham, P.W. and McMahon, A.P. 2001. Hedgehog signaling in animal development: Paradigms and principles. Genes \& Dev. 15: 3059-3087.

Jia, J., Amanai, K., Wang, G., Tang, J., Wang, B., and Jiang, J. 2002. Shaggy/GSK3 antagonizes Hedgehog signalling by regulating Cubitus interruptus. Nature 416: 548-552.

Jiang, J. 2002. Degrading Ci: Who is Cul-pable? Genes \& Dev. 16: $2315-2321$.

Jiang, J. and Struhl, G. 1995. Protein kinase A and Hedgehog signalling in Drosophila limb development. Cell 80: 563572.

- 1998. Regulation of the Hedgehog and Wingless signalling pathways by the F-box/WD40-repeat protein Slimb. Nature 391: 493-496.

Lecuit, T., Brook, W.J., Ng, M., Callega, M., Sun, H., and Cohen, S.M. 1996. Two distinct mechanisms for long-range patterning by Decapentaplegic in the Drosophila wing. Nature 381: 387-393.

Mao, J., Wang, J., Liu, B., Pan, W., Far III, G.H., Flynn, C., Yuan, H., Takada, S., Kimelman, D., Li, L., et al. 2001. Low-density lipoprotein receptor-related protein-5 binds to Axin and regulates the canonical Wnt signaling pathway. Mol. Cell 7: 801-809.

Methot, N. and Basler, K. 1999. Hedgehog controls limb development by regulating the activities of distinct transcriptional activator and repressor forms of Cubitus interruptus. Cell 96: 819-831.

- 2000. Suppressor of Fused opposes Hedgehog signal transduction by impeding nuclear accumulation of the activator form of Cubitus interruptus. Development 127: 40014010.

Monnier, V., Ho, K.S., Sanial, M., Scott, M.P., and Plessis, A. 2002. Hedgehog signal transduction proteins: Contacts of the Fused kinase and $\mathrm{Ci}$ transcription factor with the Kinesin-related protein Costal2. BMC Dev. Biol. 2: 4.

Motzny, C.K. and Holmgren, R. 1995. The Drosophila cubitus interruptus protein and its role in the wingless and hedgehog signal transduction pathways. Mech. Dev. 52: 137-150.

Murone, M., Rosenthal, A., and de Sauvage, F.J. 1999. Sonic hedgehog signaling by the patched-smoothened receptor complex. Curr. Biol. 9: 76-84.

Nellen, D., Burke, R., Struhl, G., and Basler, K. 1996. Direct and long-range action of a DPP morphogen gradient. Cell 85: 357-368.

Ohlmeyer, J.T. and Kalderon, D. 1997. Dual pathways for induction of wingless expression by protein kinase A and Hedgehog in Drosophila embryos. Genes \& Dev. 11: 22502258. 
Jia et al.

1998. Hedgehog stimulates maturation of Cubitus interruptus into a labile transcriptional activator. Nature 396: 749-753.

Pignoni, F., Hu, B., Zavitz, K.H., Xiao, J., Garrity, P.A., and Zipursky, S.L. 1997. The eye-specification proteins So and Eya form a complex and regulate multiple steps in Drosophila eye development. Cell 91: 881-891; erratum. 1998. Cell 92: 585.

Preat, T. 1992. Characterization of Suppressor of fused, a complete suppressor of the fused segment polarity gene of Drosophila melanogaster. Genetics 132: 725-736.

Price, M.A. and Kalderon, D. 1999. Proteolysis of cubitus interruptus in Drosophila requires phosphorylation by protein kinase A. Development 126: 4331-4339.

- 2002. Proteolysis of the Hedgehog signaling effector $\mathrm{Cu}-$ bitus interruptus requires phosphorylation by glycogen synthase kinase 3 and casein kinase 1. Cell 108: 823-835.

Robbins, D.J., Nybakken, K.E., Kobayashi, R., Sisson, J.C., Bishop, J.M., and Therond, P.P. 1997. Hedgehog elicits signal transduction by means of a large complex containing the kinesin-related protein costal2. Cell 90: 225-234.

Schlessinger, J. 2000. Cell signaling by receptor tyrosine kinases. Cell 103: 211-225.

Sisson, J.C., Ho, K.S., Suyama, K., and Scott, M.P. 1997. Costal2, a novel kinesin-related protein in the Hedgehog signaling pathway. Cell 90: 235-245.

Stegman, M.A., Vallance, J.E., Elangovan, G., Sosinski, J., Cheng, Y., and Robbins, D.J. 2000. Identification of a tetrameric hedgehog signaling complex. J. Biol. Chem. 275: 21809-21812.

Stone, D.M., Hynes, M., Armanini, M., Swanson, T.A., Gu, Q., Johnson, R.L., Scott, M.P., Pennica, D., Goddard, A., Phillips, H., et al. 1996. The tumour-suppressor gene patched encodes a candidate receptor for Sonic hedgehog. Nature 384: 129-134.

Strigini, M. and Cohen, S.M. 1997. A Hedgehog activity gradient contributes to AP axial patterning of the Drosophila wing. Development 124: 4697-4705.

Struhl, G. and Adachi, A. 1998. Nuclear access and action of notch in vivo. Cell 93: 649-660.

Tabata, T. and Kornberg, T.B. 1994. Hedgehog is a signaling protein with a key role in patterning Drosophila imaginal discs. Cell 76: 89-102.

Taipale, J. and Beachy, P.A. 2001. The Hedgehog and Wnt signalling pathways in cancer. Nature 411: 349-354.

van-den-Heuval, M. and Ingham, P.W. 1996. smoothened encodes a receptor-like serpentine protein required for hedgehog signalling. Nature 382: 547-551.

Vegh, M. and Basler, K. 2003. A genetic screen for hedgehog targets involved in the maintenance of the Drosophila anteroposterior compartment boundary. Genetics 163: 14271438.

Villavicencio, E.H., Walterhouse, D.O., and Iannaccone, P.M. 2000. The Sonic hedgehog-Patched-gli pathway in human development and disease. Am. J. Hum. Genet. 67: 10471054.

Wang, Q.T. and Holmgren, R.A. 1999. The subcellular localization and activity of Drosophila cubitus interruptus are regulated at multiple levels. Development 126: 5097-5106. . 2000. Nuclear import of cubitus interruptus is regulated by hedgehog via a mechanism distinct from Ci stabilization and Ci activation. Development 127: 3131-3139.

Wang, G., Wang, B., and Jiang, J. 1999. Protein kinase A antagonizes Hedgehog signaling by regulating both the activator and repressor forms of Cubitus interruptus. Genes \& Dev. 13: $2828-2837$.
Wang, G., Amanai, K., Wang, B., and Jiang, J. 2000. Interactions with Costal2 and suppressor of fused regulate nuclear translocation and activity of cubitus interruptus. Genes \& Dev. 14: 2893-2905.

$\mathrm{Xu}, \mathrm{T}$. and Rubin, G.M. 1993. Analysis of genetic mosaics in developing and adult Drosophila tissues. Development 117: $1223-1237$.

Zhu, A.J., Zheng, L., Suyama, K., and Scott, M.P. 2003. Altered localization of Drosophila Smoothened protein activates Hedgehog signal transduction. Genes \& Dev. 17: 1240-1252. 


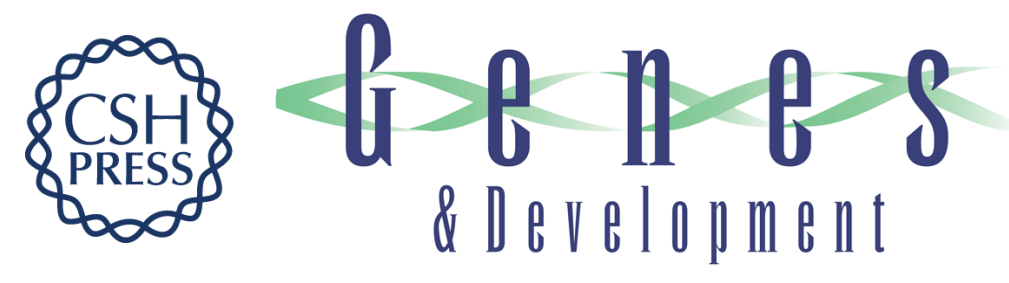

\section{Smoothened transduces Hedgehog signal by physically interacting with Costal2/Fused complex through its C-terminal tail}

Jianhang Jia, Chao Tong and Jin Jiang

Genes Dev. 2003, 17:

Access the most recent version at doi:10.1101/gad.1136603

Supplemental http://genesdev.cshlp.org/content/suppl/2003/10/09/17.21.2709.DC1
Material

References This article cites 49 articles, 22 of which can be accessed free at:

http://genesdev.cshlp.org/content/17/21/2709.full.html\#ref-list-1

License

Email Alerting Receive free email alerts when new articles cite this article - sign up in the box at the top

Service

right corner of the article or click here.

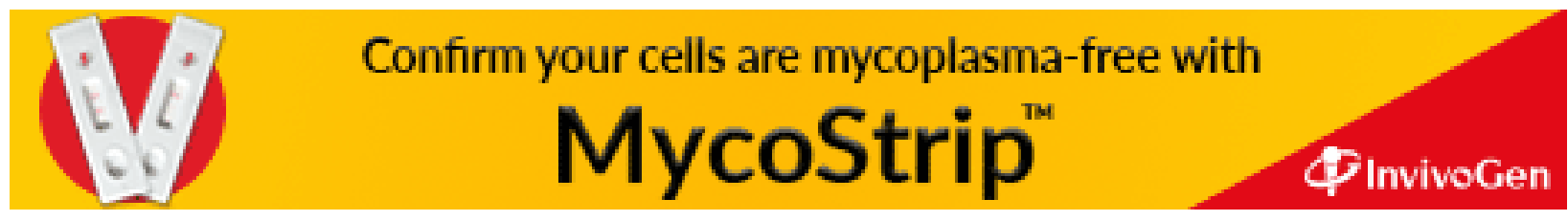

